\title{
EFICIENCIA Y MODELO DE VIBRACIONES EN MÁQUINA TURBOGENEDOR
}

\section{EFFICIENCY AND MODEL OF VIBRATIONS IN A TURBOGEN MACHINE}

\author{
MSc. Jhan Piero Rojas Suarez *, PhD. Zulmary Carolina Nieto ** \\ PhD. Mawency Vergel Ortega * \\ * Universidad Francisco de Paula Santander, Facultad de Ingeniería, \\ Grupo de Investigación \\ Euler-Graunt. \\ Av. Gran Colombia 12E-96 Colsag, Cúcuta, Norte de Santander, \\ Colombia. \\ +5775747810. \\ E-mail: \{jhanpierorojas@ufps.edu.co,mawencyvergel@ufps.edu.co\}. \\ ** Universidad de Santander, Facultad de Ciencias de la Educación, \\ Grupo de Investigación Fenix \\ Urbanización El Bosque, Avenida 4 10N-61, Cúcuta, Norte de Santander, \\ Colombia. \\ PBX 57-7-5791008 :: 57-7-5790311 \\ Zul.nieto@mail.udes.edu.co
}

Resumen: La investigación analiza la resonancia sub síncrona en un sistema de energía eléctrica, abordando este problema desde el estudio de sus causas primarias hasta el diseño de una solución general. Desde un enfoque cuantitativo, los datos de vibración recogidos tanto de forma estable como transitoria durante el arranque de los compresores tienen como objetivo generar curvas de órbita y modelos matemáticos predictivos y preventivos. Los resultados muestran disminución de consumo para condiciones iguales de temperatura y velocidad, en vibración las características de órbitas truncadas de la fricción interna relacionadas con el aumento de la velocidad, la cascada de espectro completo se construye a partir de dos transductores; se concluye que operar la torre de enfriamiento con dos bombas de recirculación con los ventiladores a igual velocidad, reduce pérdidas por consumo eléctrico, la vibración tanto en modo estacionario como transitorio genera modelos predictivos para la prevención del deterioro del rotor y la inestabilidad del compresor

Palabras clave: modelo, análisis matemático, vibraciones, visualización.

\begin{abstract}
The research analyzes sub-synchronous resonance in an electric power system, addressing this problem from the study of its primary causes to the design of a general solution. From a quantitative approach, the vibration data collected both stably and transiently during compressor start-up are intended to generate orbit curves and predictive and preventive mathematical models. The results show decreased consumption for equal conditions of temperature and speed, in vibration the characteristics of truncated orbits of internal friction related to increased speed, the cascade of full spectrum is built from two transducers, it is concluded that operating the cooling tower with two recirculation pumps with fans at equal speed, reduces losses from electricity consumption, vibration in both stationary and transient mode generates predictive models for the prevention of rotor deterioration and compressor instability
\end{abstract}

Keywords: modeling, mathematical analysis, vibrations, visualization. 


\section{INTRODUCCIÓN}

En una turbina, el vapor entra por las toberas y se expansiona hasta una presión más pequeña, al hacerlo el vapor gana velocidad a medida que el fluido avanza a través de la tobera; no hay trabajo sobre el fluido, pero si aumento de la energía cinética del mismo. Parte de la energía cinética del vapor es cedida a los alabes de la turbina, debida a la cantidad de movimiento del vapor al pasar a través de los canales de los alabes (Anderson y Van Ness, 1990). De esta forma la entalpía se convierte en energía cinética a medida que el vapor circula por la tobera una torre de enfriamiento, se reduce la temperatura del agua de enfriamiento, aproximadamente un $40 \%$ de la energía total, contenida en el combustible se pierde (Beltrán, 2012); (L Tangarife et al., 2017).

Cuando se utiliza una torre de enfriamiento, en un ciclo de vapor, el sistema, puede beneficiarse de la baja temperatura del agua de enfriamiento, ya que mejoraría las condiciones de expansión del vapor en el condensador, con el consiguiente aumento de energía disponible en la turbina. Sin embargo, hay un límite más allá del cual solo se desperdiciaría la energía, un objetivo de la investigación fue reducir el consumo eléctrico de auxiliares de la torre de enfriamiento de un turbogenerador, evaluar las pérdidas por consumo eléctrico y la eficiencia de un turbogenerador de condensación.

Una unidad turbogenerador es un generador eléctrico de bajo número de polos o de velocidad de rotación elevada (3000 rpm o más) que normalmente es impulsado por turbina de vapor o de gas (Anderson y Van Ness, 1990), en la investigación, un reductor de velocidad de caja de cambios que opera con fuel oil y suministra al sistema eléctrico de las regiones del Amazonas en el Puerto Ayacucho-Colombia, estación de 12 Megavatios (MW) de potencia (Beltrán, 2012), posee un sistema de 7 válvulas de admission de vapor, una etapa Curtis, y 30 pares de reacción, trabaja a una presión de vapor de entrada de 60 $\mathrm{Kg} / \mathrm{cm} 2$, y una temperatura de vapor de entrada de $482{ }^{\circ} \mathrm{C} ; 3$ extracciones de vapor alimentan 3 calentadores de agua, la primera extracción trabaja a una presión absoluta de $0.69 \mathrm{Kg} / \mathrm{cm} 2$ absoluto, y una temperatura de $95^{\circ} \mathrm{C}$, la segunda extracción trabaja a $1.76 \mathrm{Kg} / \mathrm{cm} 2$ con $185{ }^{\circ} \mathrm{C}$ y la tercera extracción trabaja a una presión de $6.05 \mathrm{Kg} / \mathrm{cm} 2$ y
$268{ }^{\circ} \mathrm{C}$ (Beltrán, 2012), mueve un generador síncrono de $25 \mathrm{MW}$, con una salida de $13.8 \mathrm{kV}$, y que es enfriado por hidrógeno.

El sistema de enfriamiento es proporcionado por una torre de enfriamiento con una capacidad de flujo de 24,500 galones por minuto de agua. Posee dos ventiladores con motor de capacidad de 250 HP cada uno y dos bombas con motores de capacidad de 600 HP cada una. La turbina es alimentada por una caldera tipo acuatubular, con una capacidad máxima de 104,308 kilogramos de vapor por hora, trabaja a una temperatura de $504^{\circ} \mathrm{C}$ y a una presión de $61.1 \mathrm{Kg} / \mathrm{cm} 2$, el combustible utilizado es bunker C. (Beltrán, 2012); (J Plaza, M Núñez, 2017).

En el análisis de vibración, los gráficos de base de tiempo de órbita se utilizan para determinar el ángulo de desfase, confirmar los valores de amplitud global, la frecuencia y la forma de movimiento dinámico del rotor. La interpretación de este tipo de gráficos proporciona una imagen detallada de la naturaleza de la vibración, es decir, la presencia de precarga en estado estacionario o estacionario, o si el movimiento dinámico de la línea central del eje es precesión hacia adelante o hacia atrás; (A Niño, 2018).

La capacidad de potencia suministrada por los turbogeneradores es actualmente de $38.312 \mathrm{MW}$ en el sistema, lo que representa el $74.97 \%$ de la potencia total suministrada al sistema (Moreno y Gómez, 2017). El espectro completo es una mejora del gráfico producido utilizando la forma de onda de ambos transductores XY para calcular la amplitud de ambas componentes de la frecuencia inversa y directa (Chapman, 2005); (O Suarez at el, 2018).

El vector de vibración 1X (ángulo de desfase y amplitud) en función de la rotación del eje (Chapman, 2005) y en eje Y representa el ángulo de retardo de fase de la vibración $1 \mathrm{X}$, y el eje $\mathrm{Y}$ en la gráfica inferior representa la amplitud 1X. El eje común $\mathrm{X}$ representa la rotación del eje (Rivera, 2016). Este formato en representación polar es la amplitud filtrada de la vibración del eje (1X, $2 \mathrm{X}$, nX...) y el desfase de un solo canal de vibración en función de la velocidad de rotación. Los datos utilizados en este gráfico son idénticos a los utilizados en el gráfico de bits (Dermikan, 2011). El diagrama polar se genera normalmente durante el arranque o la parada del transitorio de la máquina 


\section{METODO}

Esta investigación forma parte del paradigma cuantitativo (Ortega, Vergel y Rojas, 2019), parte de la información obtenida de bases de datos gratuitas de diferentes agencias y basada en ella

ajusta modelos estadísticos asociados a series temporales. Las bombas centrífugas, combinan el máximo rendimiento en la transmisión de energía con la simplicidad, compacidad, movimiento de rotación equilibrado, ausencia de válvulas y émbolos, descarga uniforme, servicio seguro y poco peso (Ríos, Aristizabal y Escobar, 2012). Las pruebas en las bombas de recirculación de la torre de enfriamiento, consistieron básicamente en trabajar con dos bombas a igual velocidad, y la otra prueba en trabajar con una sola bomba, el objetivo de esta prueba es reducir el consumo eléctrico de auxiliares, asumiendo que a igual velocidad el consumo total de las bombas sería menor (Rizwan, 2010).

El principio en que se basó para plantear la hipótesis son las curvas características de velocidad variable para una bomba centrífuga, en donde se determinó que al trabajar a mayor velocidad una bomba el consumo eléctrico del motor que la mueve aumenta en proporción cúbica. determinar el consumo eléctrico en los motores de la torre de enfriamiento, específicamente en los ventiladores, para condiciones establecidas de operación, con el fin de poder comparar con la operación a igual velocidad en los ventiladores. iguales condiciones de temperatura ambiente, temperatura de agua de enfriamiento en la entrada y salida de la torre. Para esta prueba se mantuvo la carga del turbogenerador constante, el vacío en el condensador se mantendrá en 82.75 milímetros de mercurio absoluto, controladas por el flujo de agua de las bombas de recirculación las cuales trabajaron a igual velocidad (Rizwan, 2010).

La temperatura del agua de enfriamiento estará regulada por los ventiladores, un ventilador trabajara a máxima velocidad y un segundo ventilador el cual trabajó a la velocidad que sea necesario para mantener la temperatura del agua al valor deseado. Las condiciones de temperatura y presión de vapor se mantendrán constantes, así como también el flujo de vapor necesario para mantener la carga deseada (Ortega, Vergel y Rojas, 2019). Se realiza prueba de operación con una bomba de recirculación variando la velocidad de la bomba para ir obteniendo diferentes contrapresiones en el condensador (Ortega, Vergel y Rojas, 2019).

Una vez se analizan valores, se realiza prueba de operación con dos bombas de recirculación a igual velocidad prueba de operación de velocidad bombas contra velocidad ventiladores variar el flujo de agua hacia el condensador de la turbina,

variando la velocidad de las bombas de recirculación en la torre de enfriamiento, con el fin de ir obteniendo diferente vacío en el condensador de la turbina. Para verificar la repetibilidad de los vectores de vibración $1 \mathrm{X}$ (Fig. 1, diagrama con sensor de localización en tren de máquina), se añadió una señal de fase temporal en el eje de baja velocidad.

El sistema de proximidad se fijó a la caja que cubre el excitador mediante adhesivo, se instaló una pieza metálica de un espesor necesario para generar de un diferencial en voltaje para generar un pulso en el sensor (Ortega, Vergel y Rojas, 2019). Se analizó el fenómeno del aumento de la vibración en el generador durante las visitas a la planta (Rizwan, 2010).

\section{RESULTADOS}

Las curvas características de velocidad variable de una bomba centrífuga (Fig. 2, curva característica de velocidad variable para una bomba centrífuga), se observó que el caudal de agua es directamente proporcional a la velocidad, la presión al cuadrado de la velocidad, y la potencia absorbida al cubo de la velocidad. Según esto doblando la velocidad del rodete impulsor de una bomba se dobla su caudal, produce una presión cuatro veces mayor y absorbe una potencia ocho veces más grande. En este caso posible operar con un $60 \%$ del flujo con el $13 \%$ de la potencia requerida para un $100 \%$ del flujo (Fig. 3 , consumo de energía eléctrica en prueba de bombas de recirculación).

Al vincular al sistema otra bomba que trabaje con igual velocidad, con temperatura del agua de salida de la torre igual en ambas pruebas, el consumo eléctrico se redujo, conforme se varía el vacío en el condensador, al variar el flujo de agua, el consumo eléctrico aumenta, el consumo eléctrico es menor cuando se trabaja con dos bombas, y la diferencia entre ambos consumos aumentó; al aumentar el flujo de agua, las bombas deben acelerarse y con una sola bomba la velocidad necesaria para mantener el flujo de agua, hace que el consumo eléctrico aumente. 
Se observó en los diferentes datos de presión y temperatura del vapor a la salida de la turbina (Tabla 1), por ejemplo para un vacío de 77.6 milímetros de mercurio absoluto y $45^{\circ} \mathrm{C}$ se tiene una entalpía del vapor de $2586 \mathrm{KJ} / \mathrm{kg}$, mientras que para un vacío de 119 milímetros de mercurio absoluto y $54^{\circ} \mathrm{C}$ se tiene una entalpía del vapor de

$2601 \mathrm{KJ} / \mathrm{kg}$, como se puede observar entre mayor es la contrapresión y la temperatura del vapor a la salida, mayor es la energía que aún está disponible en el vapor a la salida de la turbina, lo que indica que el vapor está saliendo con energía que pudo haber sido aprovechado en la turbina para realizar trabajo.

Asumiendo una operación con un vacío en el condensador de 77.6 milímetros de mercurio absoluto, punto de óptima eficiencia. El ahorro de energía en las pruebas de operación de los ventiladores de la torre de enfriamiento, mostró un ahorro promedio de $67 \mathrm{kWh}$, durante un día de operación al operar los ventiladores a igual velocidad en lugar de operar en doble rampa. operando los ventiladores a una velocidad más baja, se ahorra energía, aun y cuando se tenga que trabajar con las bombas de recirculación más aceleradas para mantener el vacío en el condensador. para un vacío de 77.6 milímetros de mercurio absoluto se tuvo un ahorro de $114 \mathrm{kWh}$. En la prueba para determinar el punto de óptima eficiencia, se logró un ahorro de energía por reducir las pérdidas al mínimo, cambiar el vacío de operación en el condensador de 87.9 a 77.6 milímetros de mercurio absoluto, se ahorra 135 $\mathrm{kWh}$. La pérdida por consumo sigue una función logarítmica, explicando el vacío un $88 \%$ de la pérdida (Fig. 4).

En las parcelas se observó el aumento progresivo de las amplitudes de vibración con el cambio de fase (Breyfogle, 2003), con la unidad a una velocidad constante (Tabla 2) El comportamiento se observó en los siguientes arranques. En todos los casos el aumento de vibración de $1 \mathrm{X}$ fue en ambos rodamientos. Teniendo en cuenta que durante las anteriores puestas en marcha la unidad no tiene un indicador de fase en el eje de baja velocidad (el indicador de fase se instaló en el eje de alta velocidad y se procesó para obtener un indicador de fase para el eje de baja velocidad), se decidió instalar una referencia de fase en el eje de baja velocidad en el tren de máquinas (Kundur, 1993).
Con la señal de fase procesada no fue posible verificar la repetibilidad de los vectores de vibración $1 \mathrm{X}$ entre el inicio y el inicio. Con el fin de descartar o confirmar un problema eléctrico del generador del rotor, durante esta nueva puesta en marcha se operó la unidad durante un tiempo determinado sin excitación ni sincronización (Peláez, 2013). El cursor de las gráficas indicaba el instante en que la unidad está sincronizada; el aumento de la amplitud de vibración y el cambio

de fase se observaron principalmente en el lado del excitador del cojinete (Pande y Newman, 2012).

En el análisis de vibration Datos de vibración utilizando la interfaz Adre 408 Sxp y las señales capturadas del sistema de monitorización y protección 3500 (Moreno y Gómez, 2017). Adicionalmente, a la instalación existente, se le agregó un indicador de fase al eje de baja velocidad. Esta señal se envió al rack existente y se agregó al módulo de fasores clave correspondiente. Se incluyeron los valores de vibración obtenidos en condiciones de carga generando a 15 MW. Amplitudes de los valores globales en cada rodamiento radial en el tren de máquinas los mayores valores observados corresponden a los rodamientos 4 y 8 (Tabla 2). Detalle de estas cuatro señales es el caso del rodamiento 8, puntos VT7719A y VT-7719B, el componente de vibración $1 \mathrm{X}$ corresponde aproximadamente al $90 \%$ del valor global o directo, pero en el rodamiento 4, puntos VT-7715A y VT-7715B el valor correspondiente a la velocidad de rotación sólo alcanza el $8 \%$ del valor global. En las siguientes páginas de este artículo se analizará por qué la diferencia entre el valor global y el valor $1 \mathrm{X}$ correspondiente a la marcación 4.

De acuerdo con los valores obtenidos en la tabla 1, la máquina está operando bajo los límites determinados por el fabricante y bajo el límite sugerido por la norma ISO 7919-2 turbina de vapor terrestre y generador de más de $50 \mathrm{MW}$ con velocidades normales de funcionamiento de 1500 rpm. $1800 \mathrm{rpm}, 3000 \mathrm{rpm}$ y $3600 \mathrm{rpm}$ (Moreno y Gomez, 2017), incluso cuando la potencia de este equipo es de $30 \mathrm{MW}$, está homologado para este estándar. Los valores predeterminados por el fabricante inicialmente para el generador fueron 80 micras por pp para alerta y 100 micras por pp para disparo, valores que fueron modificados a 150 micras por pp para alarma y 170 micras por pp para disparo. Estos fueron los últimos valores que se encontraron configurados en el sistema de monitorización y protección de la serie 3500 de 
Bently Nevada. De acuerdo a esto, se corrigió la configuración en el BN 3500 Rack (Tabla 3), y se crea un esquema con la distribución real del sensor en el tren de máquinas (Fig. 1).

Sobre la diferencia existente entre el componente de rotación $1 \mathrm{X}$ y el valor global o directo en el cojinete \#4, los puntos VT-7715A y VT-7715B, la figura 1 muestra la órbita directa correspondiente a este cojinete. En esta órbita se observa una forma inusual, que no corresponde a una trayectoria de

traslación de una línea central del eje. Los cambios repentinos observados son causados por marcas o arañazos en la superficie observados por los sensores de proximidad, este fenómeno se conoce como runout o glitch o scratch. Si bien esto no representa un peligro para el rotor, aumenta la vibración global medida por el sistema de protección de la maquinaria, sobreprotegiendo la máquina.

Se observa una ampliación de la forma de onda anterior donde la amplitud directa de la señal de vibración considera que los pulsos generados por la runout son mayores que la vibración real del eje. Este no supera las 10 micras pp en relación con las 72.8 micras pp observadas por el sensor VT-7715B (Fig. 5). El mismo tipo de runout se observa en el cojinete \#3, en otro lugar, la amplitud de los pulsos producidos por runout es menor que el caso observado en el cojinete 4, incluso es mayor que el pico de amplitud al pico de desplazamiento del eje. Las amplitudes globales con y sin el efecto de los pulsos son de 27 y 10 micrones pp respectivamente

Mientras que los cambios en los vectores de vibración $1 \mathrm{X}$ podrían ser normales durante la variación de la rotación del eje (arranque y parada), en este caso, se pueden observar las variaciones ocurridas durante la velocidad constante en el rango de 1430 - $1490 \mathrm{rpm}$. El hecho de que estas variaciones ocurrieran cuando la máquina no estaba excitada o sincronizada, confirma que la naturaleza de este comportamiento tuvo su origen en el movimiento o fenómeno de naturaleza mecánica no eléctrica (Peláez, 2013).

Se podría inferir que los cambios que continúan ocurriendo durante la operación de carga, son de origen mecánico. Los mismos datos de la componente $1 \mathrm{X}$ en el diagrama polar, desde 0 hasta el instante anterior a la parada de la máquina, para el lado del excitador del rodamiento. En este gráfico, la traza roja correspondiente al arranque en sí misma con rpm crecientes hasta alcanzar los 1430 rpm (Fig. 6).

La traza correspondiente a la condición de no carga, a velocidad constante, aumentando ligeramente hasta $1490 \mathrm{rpm}$ y finalmente la traza roja correspondiente a la operación con carga. Los grandes cambios tanto en amplitud como en fase con el equipo funcionando a velocidad constante representan un comportamiento anormal para el equipo rotatorio en general. Otro aspecto del comportamiento observado, después de cada parada el rotor permanece arqueado y recupera su

forma original después de haber estado en marcha. Para secuencias de órbitas $1 \mathrm{X}$ generadas a $110 \mathrm{rpm}$ por runout en un arranque, la parada correspondiente y un nuevo arranque una hora más tarde, son aproximadamente las mismas, sólo porque en el último arranque el rotor se endereza. En condiciones normales, estas órbitas deben mantener sus formas y amplitudes casi constantes si se toma la misma velocidad de balanceo lenta, debido a que el runout debe ser constante. En este caso, la desviación variable es causada por el efecto térmico.

El comportamiento observado muestra un tipo particular de frotamiento sincrónico, cuando el componente principal de la vibración es $1 \mathrm{X}$ y el rotor entra en contacto con la parte estacionaria, este contacto se produce en el mismo punto de la superficie durante las sucesivas revoluciones. Este contacto producirá una zona de calor para el efecto de fricción que tiende a flexionar el rotor en esta dirección para la dilatación axial de esta zona. Esta flexión térmica influye directamente en el componente $1 \mathrm{X}$ de vibración de un rotor, y genera un nuevo deporte pesado (generado por el rotor excéntrico) que se combinará con el punto pesado original del rotor provocando un nuevo punto pesado que es la combinación de estos dos puntos pesados en una posición angular diferente. El hecho de modificar la posición angular del punto pesado hace que el vector de vibración se modifique por la misma magnitud (explicado por el modelo matemático de respuesta síncrona del rotor).

En la práctica el fenómeno se observa como un vector $1 \mathrm{X}$ que sigue cambiando amplitudes $\mathrm{y} / \mathrm{o}$ fase. Debido a que es un generador, los rodamientos y los retenes constituyen la zona más probable para producir fricción. La inspección de los rodamientos y la operación en posición radial dentro de la holgura del rodamiento descartan el 
roce del rodamiento. Esto deja la probabilidad de rozamiento contra el retén de aceite. Como el roce es una consecuencia de cualquier otro fallo y no un fallo en sí mismo, debido a la excentricidad entre el retén de aceite y el rodamiento o por un juego de sellado inadecuado. Se debe tener en cuenta que el juego del retén de aceite debe ser como mínimo el mismo juego del rodamiento. Por último, teniendo en cuenta que la última puesta en marcha, el aumento de la amplitud de vibración y el cambio de fase es mayor en el rodamiento del excitador es muy probable que la ubicación de la fricción sea de nuevo el sello de aceite en este rodamiento.

Los datos recogidos muestran una tendencia a la estabilización de la luz, con valores de vibración en un componente $1 \mathrm{X}$ de 60 micrones' pp en contraste con los 95 micrones registrados en el primer arranque. Esto podría ser el resultado del desgaste del material durante la condición de roce y aumentar la holgura del sello de aceite y luego el roce desaparecería con todos los síntomas que causó.

Adicionalmente, a las lecturas de las sondas de proximidad, la vibración en aceleración fue registrada en forma discreta en los rodamientos de entrada y salida de la caja de cambios (rodamientos 3 y 6) en diferentes condiciones de carga, utilizando un colector de datos portátil. Los puntos de medición se indican en color rojo (Fig. 7). Los valores obtenidos se compararon con el estándar internacional ansi/agma 6000-B69. En base a esta norma, todos los componentes del engranaje están por debajo del valor máximo recomendado para especificar en todos los casos el componente de frecuencia de cada componente de la caja de cambios.

Se recomienda realizar la verificación de holgura durante el montaje del sello del cojinete en ambos cojinetes del generador (Pande y Newman, 2012), incluyendo la holgura efectiva entre éstos y el rotor con el fin de asegurar el libre movimiento del rotor. Si el comportamiento anormal durante la última puesta en marcha se observó en el lado del excitador (Casanova, 2005), durante las anteriores puestas en marcha se observó en ambos rodamientos. Por esta razón, esta recomendación incluye ambos rodamientos (Yu, 2010). Sugerimos realizar una recuperación de la superficie y eliminación de arañazos del eje en el área de medición tanto en los cojinetes 3 y 4 del reductor de velocidad (Moreno y Gómez, 2017). Sugerir la inclusión de un sensor de fase en el montaje permanente en el eje de baja velocidad del tren de máquinas (Moosa y Sajid, 2010).

\section{CONCLUSIONES}

Operar la torre de enfriamiento con dos bombas de recirculación con los ventiladores a igual velocidad, reduce pérdidas por consumo eléctrico, donde el punto de óptima eficiencia con un vacío en el condensador de 77.6 milímetros de mercurio con un mayor ahorro de energía. Las amplitudes de vibración excesivas experimentadas por el expansor se debieron al proceso y no a la máquina en sí, que consiste en la acumulación de depósitos de catalizador tanto en los componentes

estacionarios como en los giratorios, lo que provoca el desequilibrio de la masa del rotor y el aumento de la amplitud síncrona o $1 \mathrm{X}$ cuando parte de estos depósitos se desprenden. Los niveles reales de vibración en el tren de generación están por debajo de los límites sugeridos por la Norma Internacional ISO 7919-2, y también por debajo de los niveles de alarma y disparo autorizados por el fabricante. Los niveles de vibración relativamente altos obtenidos en los rodamientos 3 y 4 de la caja de cambios son causados por la salida mecánica debido a las imperfecciones en el eje se localizan los sensores de vibración (tipo de salida de glitch) Los valores de la vibración real no superan las 10 micras pp. El comportamiento anormal en ambos cojinetes del generador, más notorio en el cojinete del excitador, fue causado por la presencia de rozamiento síncrono en los retenes de aceite de este cojinete. Teniendo en cuenta que un roce es siempre consecuencia de otro fallo y no de un fallo en sí mismo, la causa más probable de este roce podría ser la excentricidad entre el rodamiento y el retén de aceite o un juego insuficiente entre los retenes y el eje. Dependiendo de la interferencia entre ellos, el roce podría tardar más o menos tiempo en desaparecer.

\section{REFERENCIAS}

Anderson, P., Agrawal, B. and Van Ness, J. (1990). Subsynchronous resonance in power systems, IEEE, Estados Unidos.

AB Niño (2018) Micro turbina Peltón, una solución real de energía para zonas no interconectadas (ZNI). Revista Colombiana de Tecnologías de, ISSN: 1692-7257. Avanzada

Beltrán, J. (2012). Guía para una gestión basada en procesos, Instituto Andaluz de Tecnología, España. 
Moreno, A. and Gómez, C.A. (2017). “System1 helps keep operation in turboexpander at fcc plant". Orbit, Vol. 11, No. 7.

Clymer, J. R. (1992). "Discrete Event Fuzzy Airport Control”. IEEE Trans. On Systems, Man, and Cybernetics, Vol. 22, No. 2.

Chapman, S. (2005). Máquinas eléctricas. McGraw Hill, México.

Rivera Salamanca, C. (2016). Análisis de interacción torsionales subsíncronas en sistemas de potencia con compensación serie y paralelo, Universidad Nacional Autónoma de México, México.

Dermikan, H., Spoher, J. and Krishna, V. (2011). Service System Implementation. Springer Publishing, New York.

Ortega-Sierra, A., Vergel-Ortega, M. y RojasSuárez, J.P. (2019). Microenseñanza en cálculo vectorial: su impacto desde un enfoque basado en competencias, Ecoe Ediciones, Bogotá.

Ríos, C., Aristizabal, M. and Escobar, E. (2012). "Modelamiento de sistemas eléctricos en presencia de armónicos". Scientia Et Technica Vol. 22, No. 84.

Rizwan, A. (2010). Six Sigma and Developing Countries, Editorial Abdurrahman Coskun, Pakistan.

Duncan, J. and Mulukufla, S. (2012). Sistemas de potencia análisis y diseño, Editorial ciencias e ingeniería, Madrid.

Breyfogle, F. (2003). Implementing Six Sigma.Editorial John Wiley \& Sons, Hoboken.

Kundur, P. (1993). Power system stability and control, Editorial McGraw Hill, California.

Peláez, M. (2013). "Dimensions of rules and their correspondence to rule-governed behavior European Journal of Behavior Analysis". Vol. 14, No. 259

O Suarez, C Vega, E Sánchez, A Pardo. (2018) Degradación anormal de p53 e inducción de apoptosis en la red P53-mdm2 usando la estrategia de control tipo pin. Revista Colombiana de Tecnologías de Avanzada, ISSN: 1692-7257

Pande, S. and Neuman, P. (2012). Las claves prácticas de Seis Sigma Una guía dirigida a los equipos de mejora de procesos, McGraw Hill, Bogotá.

Casanova, R. (2005). Mejoramiento de los tiempos operativos basado en la metodología six sigma en Areke Energy C.A. Universidad Gran Mariscal de Ayacucho, Barcelona

L Tangarife, M Sánchez, M Rojas (2017). Modelo de interventoría de tecnologías de información en el área de conocimiento de la gestión del alcance de PMBOK® y alineado con ISO 21500 y COBIT®. Revista

Colombiana de Tecnologías de Avanzada, ISSN: 1692-7257

Yu, Y. (2010). Electric Power System Dynamics, Editorial Academic press, Estados Unidos

Moosa, K. and Sajid, A. (2010). Critical analysis of Six Sigma Implementation Total Quality Management, Vol. 21, No. 748.

JEG Plaza, MAR Nuñez, (2017) Formación en competencias específicas para la industria del software colombiano. Experiencias del uso del aprendizaje basado en proyectos. Revista Colombiana de Tecnologías de Avanzada, ISSN: 1692-7257. 